\title{
Closing a gap in tuberculosis care
}

$\mathrm{V}$ aishaly Sontake personifies failures in India's private and public health systems. The 27-year-old mother of two was initially diagnosed with tuberculosis (TB) by her private-sector family physician. After a year on treatment, paid for by her in-laws, the four-footten-inch woman weighed only 25 kilograms. She was admitted to Mumbai's public TB hospital and diagnosed with extremely drug-resistant TB (XDR-TB). After a few months of treatment, she felt better, then fell sick again. Two private practitioners refused to treat her; she ended up arriving by stretcher at the Médecins sans Frontières (MSF) clinic in Mumbai.

That was in 2012. After about eight months, the right combination of drugs to treat her XDRTB was finally found. Three months later, her sputum was negative for TB bacillus.

"Pre-MSF was a frustrating time," she said. "I was on the same pills two times, with no improvement either time. I wanted to take something that worked faster, better and with fewer side effects."

Sontake's story is all too common. Because of inadequate diagnostics, incorrect drug regimens and other factors, Indian patients with drug-resistant TB (DR-TB) typically move between private and public care, becoming increasingly impoverished and ill while spreading their disease in crowded home and work environments.

MSF's independent clinic in the suburb of Khar is addressing this gap in care. In addition, it is conducting research aimed at increasing the number of people covered, and is striving to introduce change to the public system.

MSF opened its clinic in 1999 at the invitation of the government, which wanted MSF's technical expertise for scaling up diagnostic services. Since

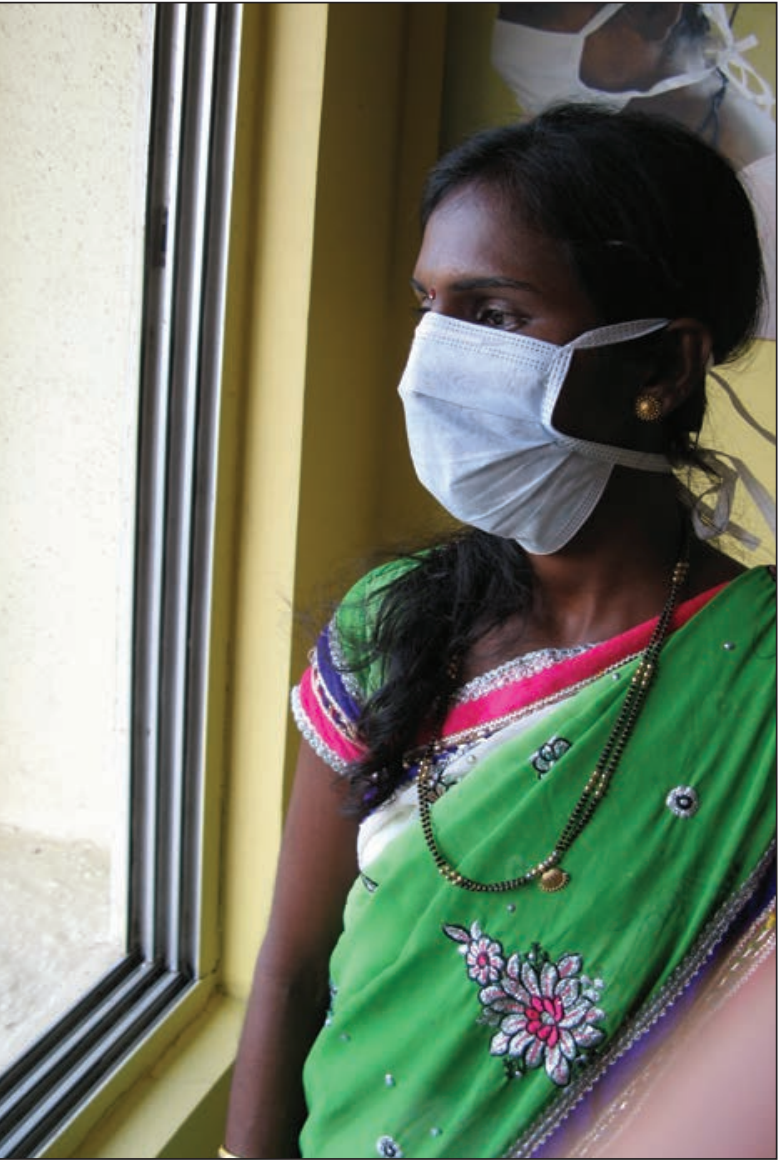

Vaishaly Sontake couldn't find the care she needed in either the public or private health system.

then, the charity has provided ambulatory medical and psychosocial care to more than 1000 people living with DR-TB, HIV, hepatitis B and C, or a combination of these diseases. Today, the clinic's 224 patients include 169 with HIV (35 on third-line antiretrovirals not available in the public system) and 55 with DR-TB (11 coinfected with HIV).

MSF has provided TB care for three decades in numerous settings, from conflict zones to urban slums; Mumbai exemplifies the latter, with $62 \%$ of its 22 million residents living in slums. And though basic TB is treated in the publicly funded health system, DR-TB is often over- or undertreated; in either case, this means the number and severity of cases increases. In 2014, Mumbai had 3522 reported cases of MDR-TB and 296 cases of XDR-TB; by 2011/12 there were 410 and 33 cases, respectively. Many, if not most, cases are not reported, however.

"It's MSF's role to take on people who are left out of the system," said the clinic's interim project coordinator, Andrews Weisz. "The ministry of health is slower than small organizations, so they're still not covering difficult lines of DR-TB."

The clinic's 53 staff -47 of whom are Indian — include two doctors, four outreach nurses, six patient-support workers and four outreach-support workers. Education and counselling are essential at the outset so patients understand the treatment and can decide whether to proceed, said Pramila Singh, MSF's clinic manager. "We have patients who have been on treatment two, three, five years. These are the cases we receive. The patients are very complex, so it's very important to explain everything to them, especially the side effects. Because they're exposed to the injections and may start to lose their hearing, some patients lose their eye sight, cannot walk ... there are a lot of side effects."

Patients come to the clinic from up to 100 kilometres away, meaning outreach is essential. MSF locates a doctor or nurse in the patient's community to provide daily injections and someone in the patient's home to report to the clinic at six-month intervals during the two-year treatment. An MSF infection-control worker assesses patients' homes, providing fans and other infection-prevention measures; some of these homes, measuring 12 by 8 feet, house 10 people, including children.

Financial supports, such as travel allowances and food rations, plus sup- 
port groups, counselling and a psychiatrist, help patients cope with the difficult treatment. In August, MSF trained its first DR-TB peer worker, who talks to fellow patients about how he managed the two-year treatment. MSF is also training patient activists to lobby the government. "HIV had great activists who changed everything. We're starting that with TB now," said Singh.

Despite MSF's efforts, not every patient is a success story. In those cases, staff are there to alleviate suffering. "That's definitely the goal for me," said Dr. Sylvie Jonckheere, the medical technical referent. "With XDR and totally drugresistant patients we can still do that. You can manage symptoms, support them psychologically and support families. Mostly we're talking about a good death and avoiding spreading infection."

Outside its clinic, MSF looks to improving DR-TB care on a broader scale. It provides guidance on infection control and counselling, plus technical medical support at Sewri TB hospital, Asia's largest with 1200 beds. In 2014, MSF trained 240 nurses and 370 housekeeping and support staff. MSF also gives technical support to the Revised National TB control Programme and Municipal Corporation of Greater Mumbai. And the collaboration works both ways. For example, MSF recently got government permission to have compassionate use of two new TB drugs: bedaquiline and delamanid. "These are still in clinical trial," said Weisz, "so we're gaining experience with these drugs. We're researching their efficacy."

\section{New project}

MSF will soon enhance this collaboration in a new project. In late February, the clinic is slated to move from the comparatively posh Khar ward to the Dharavi area, which includes one of the world's largest slums, with as many as one million residents. The move will provide many patients with easier access and allow the clinic to take part in a new MSF project to help the government set up rapid drug-sensitivity diagnostics and to correct treatment protocols for DR-TB so that they are based on drug susceptibility.

Currently, diagnostics are a problem and treatment is expensive and long with poor outcomes, said Dr. Daniel Remartinez Ferrer, MSF Belgium's medical coordinator in Delhi. "MSF and the ministry of health will be piloting a new diagnostic and treatment protocol," They will also collaborate on research. "Working within the system is more likely to make a difference and change the system."

MSF and the Public Health Department of the Municipal Corporation of Greater Mumbai signed a Memorandum of Understanding in October 2015; the new project is slated to open sometime in the first half of 2016, but details are not yet being released and the government won't comment.

Weisz said: "We're not here to replace the ministry of health. The idea is to collaborate with them to provide treatment, conference resources and urge change. We're creating a model of care that can be replicated. Through this we will demonstrate what can be done." It is a partnership, he explained. MSF will be bringing its international experience from the clinic in Mumbai: clinical management, patient support, infection control. "We are also aiming to learn from the ministry of health and strengthen the interventions in high burden areas."

The most important thing is transferring knowledge, then providing support and mentoring, adds Jonckheere. "They don't need someone by their side all the time, but in complex cases you always want to have group discussions; you can't ask one person to take responsibility for these."

"The goal is to help patients and build experience and skills that hopefully we can transfer to a public health approach on a smaller scale and that hopefully can be duplicated. You have to keep that in mind, otherwise ..." She shrugs.

The magnitude of need and the number of problems make change challenging. For example, the Indian government currently recommends rapid molecular tests using diagnostic tools like GeneXpert for initial testing only, but the molecular testing device is available at only 10 public and 8 private facilities in Mumbai. An accurate diagnosis at the outset can help ascertain drug-resistant status and determine whether first- or second-line treatment will work. "In the absence of this, people with DR-TB get first-line, which is wrong. They will not get better and will risk amplifying their resistance to other drugs," said Dr. Suvanand Sahu, deputy executive secretary of United Nations-based Stop TB partnership Secretariat.

"When you have a good test like GeneXpert, why not offer it to everyone?" he asked, then quickly answered his own question: resource limitations in the face of huge needs. India has the highest TB burden and mortality in the world: 2.3 million. A growing number — nearly 400000 in 2014 — are newly diagnosed or in retreatment for DR-TB.

But rather than stepping up support, the government cut the Revised National Tuberculosis Control Programme budget by $31 \%$ in 2014 , according to a media report. Three government officials did not respond to emailed inquiries from CMAJ.

World leaders have agreed to end TB by 2030 under the Sustainable Development Goal 3.

"It won't happen overnight," Sahu concedes. "But we know they have capacity and can progress at a much faster pace."

This can happen only with increased funding, private sector contribution and a national movement steered by Prime Minister (PM) Narendra Modi's office, with participation of the civil society and communities, says Sahu. "What works in India is a campaign approach, not routine. We've seen this with polio and the clean India campaign. It comes from the PM so it mobilizes everyone. ... That's what we need for TB. We've asked for a meeting with Modi." — Barbara Sibbald, Mumbai, India

CMAJ 2016. DOI:10.1503/cmaj.109-5238 